\title{
New symmetries in Fierz-Pauli massive gravity
}

\author{
Gianmassimo Tasinato ${ }^{1}$, Kazuya Koyama ${ }^{1}$, Gustavo Niz ${ }^{1,2}$ \\ ${ }^{1}$ Institute of Cosmology 83 Gravitation, University of Portsmouth, \\ Dennis Sciama Building, Portsmouth, PO1 3FX, United Kingdom. \\ 2 Departamento de Física, Universidad de Guanajuato, \\ DCI, Campus León, C.P. 37150, León, Guanajuato, México.
}

\begin{abstract}
We expose a new symmetry for linear perturbations around a solution of non-linear Fierz-Pauli massive gravity plus a bare cosmological constant. The cosmological constant is chosen such that the background metric is flat while the Stückelberg fields have a non-trivial profile. Around this background, at linear order the new symmetry reduces the propagating degrees of freedom to those of General Relativity, namely the massless helicity 2 modes only. We discuss the physical consequences and possible applications of these findings.
\end{abstract}

\section{Introduction}

One of the most important properties of general relativity (GR) is that it propagates two helicity-2 interacting degrees of freedom. This property is ensured by the gauge symmetry associated with general coordinate invariance, together with the particular structure of the Einstein-Hilbert action. The fact that long-range fifth forces, for example due to light scalars, are not observed in the Solar system, suggests that GR is the correct theory of gravity near the Earth. On the other hand, serious theoretical issues with Einstein gravity in the infrared, as the cosmological constant problem and the need to explain the observed current acceleration of the universe, motivate to consider alternative theories of gravity. Typically, these theories involve modifications of GR at short and/or large distances, and provide compelling explanations for the observed acceleration of the universe. However, in most cases they spoil the properties that characterize GR, such as coordinate invariance or the number of degrees of freedom. These additional degrees of freedom may have unacceptable pathologies (for example, they may be ghosts), or lead to predictions in disagreement with current observations. However, as we are going to discuss here, on some backgrounds, new gauge symmetries might emerge, which are able to remove the new undesired degrees of freedom.

One of the simplest modification of GR consists of adding a mass term for the graviton to the EinsteinHilbert (EH) action, leading to a theory of massive gravity. This possibility was proposed long time ago by Fierz and Pauli [1], who considered a mass term which is uniquely defined at linear order in perturbations around flat space. Here we consider a theory that minimally extends the Fierz-Pauli (FP) action beyond linear order in perturbations which is given by (we set $M_{p}^{2} / 2=1$ ):

$$
I=I_{E H}+I_{F P}=\int d^{4} x \sqrt{-g}[R-2 \Lambda]-\frac{m^{2}}{4} \int d^{4} x \sqrt{-g} h_{\mu \nu} h_{\rho \sigma}\left(g^{\mu \rho} g^{\nu \sigma}-g^{\mu \nu} g^{\rho \sigma}\right) .
$$


In the previous formula $g_{\mu \nu}$ is the dynamical metric in four dimensions, and $h_{\mu \nu}=g_{\mu \nu}-\eta_{\mu \nu}$ represents the displacement of the dynamical metric from a fiducial, fixed metric that we choose to be the Minkowski spacetime. Notice that we have also added a bare cosmological constant term to the usual Einstein-Hilbert piece, controlled by the parameter $\Lambda$.

In the case $\Lambda=0$, Fierz and Pauli proved that the action describing quadratic perturbations around Minkowski space contains five massless degrees of freedom: two tensor, two vectors, and one scalar [1]. Some time later, Boulware and Deser showed that an additional propagating degree of freedom, a ghost, arises when a point-like source is added to flat space, whose kinetic term is weighted by the inverse of the source mass [2. In total, around a generic background this theory propagates six degrees of freedom. This is expected since general coordinate invariance is broken by the mass term. See [3] for a recent review.

However, it is interesting to ask whether a background solution for the theory (11) exist, around which a smaller number of degrees of freedom propagate due to the emergence of new gauge symmetries. The answer is affirmative, as we will show in this work. Apart from the Minkowski spacetime, other exact vacuum solutions have been found for the action (11) with no cosmological constant [4. In this paper, we first generalize these solutions to include an arbitrary bare cosmological constant $\Lambda$, and then analyse in detail the dynamics of linear perturbations around a particularly interesting and simple configuration, in which the effect of the graviton mass and the cosmological constant terms compensate each other, and lead to a flat physical background. Around this configuration, a new gauge symmetry emerges in the quadratic action for perturbations, which removes the dynamical vector and scalar degrees of freedom, leaving the massless helicity-2 mode as the only dynamical state. Consequently, the dynamics of linearized fluctuations for this theory around our flat background behaves exactly as in GR.

Our findings explicitly show that enhanced symmetry points exist in the space of background solutions of modified gravity models, such as massive gravity. These configurations might represent examples of consistent backgrounds where we can live on, and in which the infrared issues of GR could be addressed more successfully than in GR.

The paper is organized as follows. In section 2, we discuss self-accelerating solutions in the generalised Fierz-Pauli model, including a bare cosmological constant. We show that, imposing a particular tuning of parameters, the physical metric reduces to the Minkowski spacetime. In section 3, we study linear perturbations around this non-trivial flat space solution, using the ADM formalism. We split the analysis in tensor, vector and scalar modes with respect to the spatial metric and show that it only propagates the massless tensor modes as in GR. In section 4, we explicitly construct the gauge symmetry which reduces the number of physical degrees of freedom around our specific background, and we show how to express it in a covariant way. Finally, we conclude in section 5 with a discussion of our results.

\section{New exact solutions}

In order to study the theory (11) and its exact solutions, it is convenient to implement the Stückelberg trick to recover diffeomorphism invariance following [5. This can be achieved by introducing a covariantization of the metric displacement $h_{\mu \nu}$ in terms of the following definition

$$
g_{\mu \nu}=\eta_{\mu \nu}+h_{\mu \nu}=H_{\mu \nu}+\eta_{\alpha \beta} \partial_{\mu} \phi^{\alpha} \partial_{\nu} \phi^{\beta} .
$$

Therefore, the quantity $H_{\mu \nu}$ is given by a combination of the physical metric $g_{\mu \nu}$, and a metric in field space

$$
f_{\mu \nu} \equiv \eta_{\alpha \beta} \partial_{\mu} \phi^{\alpha} \partial_{\nu} \phi^{\beta}
$$

The FP part of the Lagrangian density, in terms of the quantity $H_{\mu \nu}$, can be written as

$$
\mathcal{L}_{F P}=-\frac{m^{2}}{4} \sqrt{-g} H_{\mu \nu} H_{\rho \sigma}\left(g^{\mu \rho} g^{\nu \sigma}-g^{\mu \nu} g^{\rho \sigma}\right) .
$$


The Stückelberg fields $\phi^{\alpha}$ have been introduced to restore reparametrisation invariance, so that the previous action is invariant under $x^{\alpha} \rightarrow x^{\alpha}+\xi^{\alpha}$, provided these Stückelberg fields $\phi^{\alpha}$ transform as scalars with respect to space-time symmetries (their indexes are raised and lowered by means of the fiducial metric $\eta_{\alpha \beta}$ ). Choosing the unitary gauge, defined by $\phi^{\alpha}=x^{\alpha}$, one obtains $H_{\mu \nu}=h_{\mu \nu}$ which leads to the theory written as in eq. (1). The simplest background solution for this theory, in absence of bare cosmological constant, is Minkowski space, given by

$$
g_{\mu \nu}=\eta_{\mu \nu}, \phi^{\mu}=x^{\mu} \quad, \quad H_{\mu \nu}=0 \quad, \quad \Lambda=0 .
$$

Salam and Strathdee found other vacuum solutions, that we now generalize to the case of a non-zero cosmological constant (see Appendix $\mathrm{A}$ for a full derivation). We write them in a coordinate system that is particularly useful for studying cosmology, where the metric reduces to the flat slicing of a maximally symmetric space, namely

$$
d s^{2}=-d t^{2}+e^{2 \tilde{m} t}\left(d x_{1}^{2}+d x_{2}^{2}+d x_{3}^{2}\right)
$$

while the Stückelberg fields acquire the following non-trivial background profile

$$
\begin{aligned}
\phi^{0} & =\sqrt{\frac{3}{2}} \frac{1}{\tilde{m}}\left[\operatorname{arctanh}\left(\frac{2 \sinh \tilde{m} t+\tilde{m}^{2} r^{2} e^{\tilde{m} t}}{2 \cosh \tilde{m} t-\tilde{m}^{2} r^{2} e^{\tilde{m} t}}\right)-\tilde{m} r e^{\tilde{m} t}+\operatorname{arctanh}\left(\tilde{m} r e^{\tilde{m} t}\right)\right], \\
\phi^{i} & =\sqrt{\frac{3}{2}} e^{\tilde{m} t} x^{i} \quad(i=1,2,3) .
\end{aligned}
$$

In the previous equations we dubbed for simplicity $r \equiv \sqrt{x_{1}^{2}+x_{2}^{2}+x_{3}^{2}}$, and called

$$
\tilde{m}^{2}=\frac{m^{2}}{4}+\frac{\Lambda}{3} .
$$

Notice that the Hubble scale is set by the FP mass and the cosmological constant, thus a solution exhibits the acceleration if $\tilde{m}>0$. In absence of cosmological constant, $\Lambda=0$, and for a non-zero $m$, the solution exhibits the self-acceleration, i.e. an acceleration without cosmological constant.

The general solution discussed above admits an interesting limit leading to flat space, when choosing the cosmological constant $\Lambda=-(3 / 4) m^{2}$. It is easy to check that this background solution for the metric and Stückelberg fields reads

$$
g_{\mu \nu}=\eta_{\mu \nu} \quad, \quad \phi^{\mu}=\sqrt{\frac{3}{2}} x^{\mu} \quad, \quad H_{\mu \nu}=-\frac{1}{2} \eta_{\mu \nu} \quad, \quad \Lambda=-\frac{3}{4} m^{2} .
$$

Notice that the previous solution is different from the simplest Minkowski solution in the absence of cosmological constant term (eqs. (5)), by a constant in the Stückelberg fields. The field space metric $f_{\mu \nu}$, defined in eq. (3), retains the same symmetries of the physical space-time metric $g_{\mu \nu}$.

In the next section, we will study the dynamics of perturbations around the background of eq. (10), showing that a new emerging gauge symmetry reduces the number of degrees of freedom to the ones of pure GR with no mass term and no cosmological constant.

\section{Dynamics of perturbations}

\subsection{Definition of perturbations}

We follow an ADM approach and use Latin indexes to characterize quantities in the three spatial dimensions. The metric reads

$$
d s^{2}=-N^{2} d t^{2}+\gamma_{i j}\left(d x^{i}+N^{i} d t\right)\left(d x^{j}+N^{j} d t\right)
$$


Perturbations to the lapse function and the shift vector are defined as

$$
N=1+A, \quad N^{i}=\mathcal{B}^{i},
$$

and to the three dimensional metric as

$$
\gamma_{i j}=\delta_{i j}+2 h_{L} \delta_{i j}+2\left(\mathcal{E}_{i j}-\frac{1}{3} \delta_{i j} \mathcal{E}_{k}^{k}\right)
$$

where the curvature perturbation is defined as $\mathcal{R} \equiv h_{L}-\frac{1}{3} \mathcal{E}_{k}^{k}$. Using the extrinsic curvature $K_{i j} \equiv$ $\frac{1}{2 N}\left(\nabla_{i} N_{j}+\nabla_{j} N_{i}-\dot{\gamma}_{i j}\right)$, we express the Einstein-Hilbert part of the action (1) in the usual form

$$
I_{E H}=\int d t d^{3} x N \sqrt{\gamma}\left[R-2 \Lambda+K_{i}^{j} K_{j}^{i}-K^{2}-2 \Lambda\right] .
$$

Furthermore, it is convenient to decompose the various quantities into scalar, vector and tensors with respect to the $3 \mathrm{D}$ spatial metric as

$$
\begin{aligned}
\mathcal{B}_{i} & =S_{i}+B_{, i}, \\
\mathcal{E}_{i j} & =h_{i j}+F_{(i, j)}+E_{, i j},
\end{aligned}
$$

with $S_{i}$ and $F_{i}$ transverse vectors, and $h_{i j}$ transverse traceless tensor, namely

$$
S_{, i}^{i}=F_{, i}^{i}=h_{i}^{i}=h_{j, i}^{i}=0 .
$$

The perturbation of Stückelberg fields split as

$$
\delta \phi_{\mu}=\left(\varphi, \chi_{i}+\psi_{, i}\right)
$$

with $\chi_{, i}^{i}=0$.

\subsection{Action quadratic in perturbations}

At linear order, the action of perturbations vanishes in agreement with (10) being a solution of (1). At quadratic order in perturbations around the background solution (10), the EH contribution to the action (11) reads

$$
\begin{aligned}
I_{E H}^{\text {quad }}=\int d t d^{3} x \quad & \left\{\dot{h}_{i j} \dot{h}^{i j}+h^{i j} \Delta h_{i j}+2 \Lambda h_{i j} h^{i j}+\frac{1}{2}\left(S^{i}-\dot{F}^{i}\right)^{, j}\left(S_{i}-\dot{F}_{i}\right)_{, j}\right. \\
& -6 \dot{\mathcal{R}}^{2}+4 \dot{\mathcal{R}} \Delta(B-\dot{E})-4 A \Delta \mathcal{R}+2 \partial_{i} \mathcal{R} \partial^{i} \mathcal{R} \\
& \left.-2 \Lambda\left(3 A \mathcal{R}+\frac{3}{2} \mathcal{R}^{2}+(A+\mathcal{R}) \Delta E+\frac{1}{2}(\Delta E)^{2}-E_{, i j} E^{, i j}-F_{i, j} F^{i, j}\right)\right\},
\end{aligned}
$$

where dots represent time derivatives. The FP part is given by

$$
\begin{aligned}
I_{F P}^{q u a d}=-\frac{m^{2}}{4} \int d t d^{3} x \quad & \{\dot{\varphi}[3 \sqrt{6} A+3 \sqrt{6} \Delta E+9 \sqrt{6} \mathcal{R}-6 \Delta \psi-3 \dot{\varphi}] \\
& +A\left[-\frac{9}{2} A-3 \Delta E-9 \mathcal{R}+3 \sqrt{6} \Delta \psi\right] \\
& +\mathcal{R}\left[-21 \Delta E-\frac{63}{2} \mathcal{R}+9 \sqrt{6} \Delta \psi\right] \\
& \left.-3\left(\Delta \psi-\sqrt{\frac{3}{2}} \Delta E\right)^{2}-6 h_{i j} h^{i j}-6 F_{i, j} F^{i, j}-6 E_{, i j} E^{, i j}+3(\Delta E)^{2}\right\} .
\end{aligned}
$$


Adding these two pieces, and setting $\Lambda=-3 / 4 m^{2}$, we obtain that the tensor and vector contributions organize in such a way to become exactly the ones of pure GR in the Minkowski background, namely

$$
I_{\text {tens }, \text { vect }}^{\text {quad }}=\int d t d^{3} x\left[\dot{h}_{i j} \dot{h}^{i j}+h^{i j} \Delta h_{i j}+\frac{1}{2}\left(S^{i}-\dot{F}^{i}\right)^{, j}\left(S_{i}-\dot{F}_{i}\right)_{, j}\right] .
$$

Therefore, they behave as in pure GR, describing the propagation of helicity- 2 states. One can prove this standard fact by a Hamiltonian analysis of the action (21), as shown in detail in Appendix B,

After fixing $\Lambda=-3 / 4 m^{2}$, the Lagrangian density associated with the total scalar contribution, up to a total derivative, is

$$
\begin{aligned}
\mathcal{L}_{\text {scal }}^{\text {quad }}= & \left\{-6 \dot{\mathcal{R}}^{2}+4 \dot{\mathcal{R}} \Delta(B-\dot{E})-4 A \Delta \mathcal{R}-2 \mathcal{R} \Delta \mathcal{R}\right\} \\
& -\frac{m^{2}}{4}\{\dot{\varphi}[3 \sqrt{6} A+3 \sqrt{6} \Delta E+9 \sqrt{6} \mathcal{R}-6 \Delta \psi-3 \dot{\varphi}] \\
& +A\left[-\frac{9}{2} A-9 \Delta E-27 \mathcal{R}+3 \sqrt{6} \Delta \psi\right] \\
& \left.+\mathcal{R}\left[-27 \Delta E-\frac{81}{2} \mathcal{R}+9 \sqrt{6} \Delta \psi\right]-3\left(\Delta \psi-\sqrt{\frac{3}{2}} \Delta E\right)^{2}\right\} .
\end{aligned}
$$

\subsection{Hamiltonian analysis of the scalar sector}

It is convenient to perform a Hamiltonian analysis of this system, in order to count the number of dynamical degrees of freedom in the scalar sector described by the quadratic Lagrangian (22). We first notice that this Lagrangian does not contain time derivatives of $A, B$, and $\psi$; these quantities will then be associated with constraints. Therefore, we have in principle three dynamical scalar degrees of freedom: $\mathcal{R}, E$ and $\varphi$. The conjugate momenta associated with these dynamical variables are

$$
\begin{aligned}
\Pi^{\mathcal{R}} & =\frac{\partial \mathcal{L}_{s}}{\partial \dot{\mathcal{R}}}=4 \Delta B-4 \Delta \dot{E}-12 \dot{\mathcal{R}} \\
\Pi^{E} & =\frac{\partial \mathcal{L}_{s}}{\partial \dot{\mathcal{E}}}=-4 \Delta \dot{\mathcal{R}} \\
\Pi^{\varphi} & =\frac{\partial \mathcal{L}_{s}}{\partial \dot{\varphi}}=-\frac{3}{4} m^{2}(\sqrt{6} A+\sqrt{6} \Delta E-2 \dot{\varphi}-2 \Delta \psi+3 \sqrt{6} \mathcal{R}) .
\end{aligned}
$$

The scalar Hamiltonian is defined as

$$
\mathcal{H}_{s}=\dot{\mathcal{R}} \Pi^{\mathcal{R}}+\dot{E} \Pi^{\mathcal{E}}+\dot{\varphi} \Pi^{\varphi}-\mathcal{L}_{s}+A \mathcal{C}_{A}+B \mathcal{C}_{B}+\psi \mathcal{C}_{\psi}
$$

where we introduce the constraint $\mathcal{C}_{A}=\partial \mathcal{L}_{s} / \partial A$, and similarly for $B$ and $\psi$, so that $\mathcal{H}_{s}$ is written in the following way

$$
\mathcal{H}_{s}=\frac{1}{24}\left[9\left(\Delta^{-1} \Pi^{E}\right)^{2}-6\left(\Delta^{-1} \Pi^{E}\right) \Pi^{\mathcal{R}}+4 \Pi^{\varphi}\left(\frac{2 \Pi^{\varphi}}{m^{2}}+9 \sqrt{6} \mathcal{R}\right)+12 \Delta\left(\sqrt{6} E \Pi^{\varphi}+4 \mathcal{R}^{2}\right)\right] .
$$

The constraints read

$$
\begin{aligned}
\mathcal{C}_{A} & =-4 \Delta \mathcal{R}-\sqrt{\frac{3}{2}} \Pi^{\varphi}, \\
\mathcal{C}_{B} & =-\Pi^{E} \\
\mathcal{C}_{\psi} & =\Delta \Pi^{\varphi} .
\end{aligned}
$$


These are first class constraints, since their mutual Poisson brackets vanish:

$$
\left\{\mathcal{C}_{A}, \mathcal{C}_{B}\right\}=\left\{\mathcal{C}_{A}, \mathcal{C}_{\psi}\right\}=\left\{\mathcal{C}_{\psi}, \mathcal{C}_{B}\right\}=0
$$

Moreover, the Poisson brackets among the constraints and the Hamiltonian satisfy the following relations

$$
\begin{aligned}
\left\{\mathcal{C}_{A}, \mathcal{H}_{s}\right\} & =-\mathcal{C}_{B}, \\
\left\{\mathcal{C}_{\psi}, \mathcal{H}_{s}\right\} & =0 \\
\left\{\mathcal{C}_{B}, \mathcal{H}_{s}\right\} & =\sqrt{\frac{3}{2}} \mathcal{C}_{\psi},
\end{aligned}
$$

which imply that the constraints are preserved under time evolution. After imposing these constraints, it is straightforward to check that the Hamiltonian vanishes. Indeed three first class constraints are able to remove all the phase-space dynamical degrees of freedom. Consequently, also the scalar sector does not contain any physical degree of freedom.

To summarize, quadratic perturbations around the flat-space background (10) lead to the propagation of only helicity-2 modes, exactly as in GR.

\subsection{The new gauge symmetry}

We can understand the result of the previous section in terms of gauge symmetries holding for the action of quadratic perturbations around the background solution (10). Starting from our Hamiltonian analysis, we can implement the standard rules for obtaining the gauge symmetry associated with the first class constraints (see for example [6]). The infinitesimal transformations that leave the quadratic scalar Lagrangian (22) invariant, up to total derivatives, read

$$
\begin{aligned}
\delta \varphi & =\sqrt{\frac{3}{2}} \epsilon_{A}-\Delta \epsilon_{\psi}, \\
\delta \psi & =\dot{\epsilon}_{\psi}+\sqrt{\frac{3}{2}} \epsilon_{B}, \\
\delta \mathcal{R} & =0 \\
\delta E & =\epsilon_{B}, \\
\delta A & =\dot{\epsilon}_{A}, \\
\delta B & =\dot{\epsilon}_{B}-\epsilon_{A},
\end{aligned}
$$

for three arbitrary functions $\epsilon_{A}, \epsilon_{B}$ and $\epsilon_{\psi}$. The parameters $\epsilon_{A}$ and $\epsilon_{B}$ are associated with the diffeomorphism invariance of the FP action, once the Stückelberg fields are introduced. The symmetry associated with $\epsilon_{\psi}$, that acts only on the Stückelberg scalars, is the new symmetry.

It is not difficult to express these symmetries in a covariant way. Adding the cosmological constant contribution to the covariant FP action (4), tuning $\Lambda=-3 / 4 m^{2}$, and expanding at quadratic order in perturbations around our solution (10), one finds the following covariant Lagrangian density

$$
\mathcal{L}_{\text {quad }}^{F P+\Lambda}=\frac{9}{32} m^{2}\left(h_{\mu}^{\mu}-\sqrt{\frac{8}{3}} \partial_{\mu} \phi^{\mu}\right)^{2} .
$$

It is easy to check that this Lagrangian is invariant, at quadratic order, under the transformation

$$
\begin{aligned}
\delta \phi^{\mu} & =\delta \phi^{\mu}-\sqrt{\frac{3}{2}} \xi^{\mu}+\chi^{\mu} \quad \text { with } \quad \partial_{\mu} \chi^{\mu}=0 \\
h_{\mu \nu} & =h_{\mu \nu}-\xi_{(\mu, \nu)} .
\end{aligned}
$$


Focussing on scalar perturbations, the symmetry (42) corresponds exactly to the extra symmetry parameterized by the function $\epsilon_{\psi}$ in eqs (35) and (36). The existence of this gauge symmetry, besides the standard diffeomorphism invariance, implies that the scalar and vector degrees of freedom do not propagate around the background (10). Note that this resulting quadratic action (41) is exactly the same as a graviton mass term proportional to $\left(H_{\mu}^{\mu}\right)^{2}$. It is known that this mass term does not modify GR, i.e. it leads only to a massless transverse-traceless graviton [7, which is consistent with our result. On a different setting, it was also shown in [8] that, thanks to a new gauge symmetry, massive gravity on a de Sitter background does not propagate scalar zero modes, when the size of the cosmological constant is tuned to a precise value depending on the graviton mass.

\section{Discussion}

We constructed new solutions for a covariantized non-linear Fierz-Pauli theory of massive gravity, equipped with a cosmological constant $\Lambda$. We focused on a particularly simple configuration corresponding to Minkowski space with a non-trivial profile for the Stückelberg fields. The space-time flatness is achieved by tuning $\Lambda$ to a particular value related to the graviton mass. We then studied linear perturbations around this configuration, and showed that it only propagates the massless helicity- 2 modes, in the same way as pure GR without a cosmological constant. We interpreted this behavior as due to a new gauge symmetry acting on the Stückelberg fields, in addition to diffeomorphism invariance.

It would be important to understand whether this new symmetry is only associated with the particular set-up discussed here, or whether it arises more generally. In other non-linear massive gravity models, several examples of self-accelerating solutions have been found [9, 10, 11, 12, 13, 14, 15]. Recently, 14] found similar situations where there are no propagating scalar and vector modes around self-accelerating open-FRW solutions in the non-linear massive gravity models proposed by de Rham, Gabadadze and Tolley [16]. See also [15] for examples of self-accelerating solutions in non-linear theories of massive gravity, around which there are no additional degrees of freedom besides tensor modes. One one hand, a common feature between our solution and the set-up of [14] is that the metric in field space preserves the same symmetries as the physical metric, which in our case correspond to the fact that both are Minkowski spacetime. On the other hand, there are other self-accelerating de Sitter solutions in the same theory for which the field space metric is not invariant under same isometries as the physical metric [9, 10, 11. Those solutions are indeed similar to the ones obtained in this article, but where the cosmological constant is not tuned with the mass term to give flat space. The non-trivial profiles for the Stückelberg fields render the analysis of the dynamics of fluctuations more difficult. However, it would be interesting to study whether new symmetries also arise while considering perturbations around these backgrounds.

Finally, it is also important to explore whether the new gauge symmetry holds only at linear order in perturbations, or survive at higher orders. If the latter is the case, then these configurations may represent examples of consistent backgrounds where we can live on, and in which the infrared issues of GR could be addressed more successfully than in GR. For example, one could envisage a mechanism that dynamically tunes the graviton mass with the cosmological constant, leading to flat space also in the presence of $\Lambda$. We will investigate these interesting questions elsewhere.

\section{Acknowledgments}

GT is supported by an STFC Advanced Fellowship ST/H005498/1. KK is supported by supported by STFC grant ST/H002774/1, ERC and the Leverhulme trust. 


\section{A Derivation of the exact solutions}

We begin by choosing the gauge $\phi^{\mu}=x^{\mu}$, which implies that $f_{\mu \nu}=\eta_{\mu \nu}$. Since we are interested in static spherical solutions to the action (10), we introduce the ansatz

$$
d s^{2}=-C(r) d t^{2}+A(r) d r^{2}+2 D(r) d t d r+B(r) d \Omega^{2},
$$

where $d \Omega^{2}=d \theta^{2}+\sin ^{2} \theta d \phi^{2}$, and to simplify expressions, one can further choose to write the field space metric $f_{\mu \nu}$ in spherical coordinates as $f_{\mu \nu} d x^{\mu} d x^{\nu}=-d t^{2}+d r^{2}+r^{2} d \Omega^{2}$.

After introducing the ansatz (44) in the equations of motion derived from the action (11), one can show there is a constraint which leads to two branches of solutions: one with $D(r)=0$ and the other where $B(r)=3 r^{2} / 2$. The first branch was studied in [17, but here, we are interested in the second branch, where exact solutions were initially found by Salam and Strathdee [4. In this second class, a new constraint enforces $\Delta(r)=A(r) C(r)+D^{2}(r) \equiv \Delta_{0}=$ const, and the rest of equations of motion admit the following solution

$$
\begin{aligned}
& A(r)=\frac{3 \Delta_{0}}{2}(p(r)+\alpha+1), \quad B(r)=\frac{3}{2} r^{2}, \\
& C(r)=\frac{3 \Delta_{0}}{2}(1-p(r)), \quad D(r)=\frac{3}{2} \Delta_{0} \sqrt{p(r)(p(r)+\alpha)},
\end{aligned}
$$

where

$$
p(r)=\frac{c}{r}+\frac{2 \tilde{m}^{2} r^{2}}{3 \Delta_{0}^{3 / 2}}, \quad \alpha=\frac{4}{9 \Delta_{0}}-1,
$$

and $\tilde{m}$ is defined as in (9). $\Delta_{0}$ and $c$ are two integration constants that obey $c \geq 0$ and $0<\sqrt{\Delta_{0}} \leq 2 / 3$ for $D(r)$ to be real. In this work we only consider $c=0$ and the extremal value $\Delta_{0}=4 / 9$. For these values, the solution (45) can be re-casted in the simple FRW form of eq. (6) by the following coordinate transformation

$$
\begin{aligned}
x^{0} \equiv t & \rightarrow \sqrt{\frac{3}{2}} \frac{1}{\tilde{m}}\left[\operatorname{arctanh}\left(\frac{2 \sinh \tilde{m} t+\tilde{m}^{2} r^{2} e^{\tilde{m} t}}{2 \cosh \tilde{m} t-\tilde{m}^{2} r^{2} e^{\tilde{m} t}}\right)-\tilde{m} r e^{\tilde{m} t}+\operatorname{arctanh}\left(\tilde{m} r e^{\tilde{m} t}\right)\right], \\
x^{i} & \rightarrow \sqrt{\frac{3}{2}} e^{\tilde{m} t} x^{i} \quad(i=1,2,3) .
\end{aligned}
$$

The last transformation implies that $r \rightarrow \sqrt{\frac{3}{2}} e^{\tilde{m} t} r$, while $\theta$ and $\phi$ remain unchanged. Furthermore, the transformations change our initial gauge so that $f_{\mu \nu} \neq \eta_{\mu \nu}$, since the initial Stückelberg fields $\phi^{\mu}=x^{\mu}$ get modified to those in (7).

\section{B Hamiltonian analysis of tensor and vector degrees of freedom}

The canonical momenta associated to the tensor and vector modes in the action (21) are

$$
\begin{aligned}
& \Pi_{h}^{i j}=\frac{\delta \mathcal{L}_{\text {tens }, \text { vect }}^{\text {quad }}}{\delta \dot{h}_{i j}}=2 \dot{h}^{i j} \\
& \Pi_{F}^{i}=\frac{\delta \mathcal{L}_{\text {tens }, \text { vect }}^{\text {quad }}}{\delta \dot{F}_{i}}=\Delta\left(S^{i}-\dot{F}^{i}\right),
\end{aligned}
$$

where $\mathcal{L}_{\text {tens,vect }}^{\text {quad }}$ is associated Lagrangian density of the action (21). In momentum's language, the Lagrangian $\mathcal{L}_{\text {tens, vect }}^{\text {quad }}$ then reads

$$
I_{\text {tens }, \text { vect }}^{\text {quad }}=\int d t d^{3} x\left[\Pi_{h}^{i j} \dot{h}_{i j}+\Pi_{F}^{i} \dot{F}_{i}-\mathcal{H}_{t}-\mathcal{H}_{v}-S_{i} \Pi^{i}\right]
$$


where

$$
\mathcal{H}_{t}=\frac{1}{4} \Pi_{h}^{i j} \Pi_{i j}^{h}-h^{i j} \Delta h_{i j}, \quad \mathcal{H}_{v}=-\frac{1}{2} \Pi_{i}^{F} \Delta^{-1} \Pi_{F}^{i},
$$

with $\Delta^{-1}$ the inverse Laplace operator. Let us first consider the tensor modes, which do not have any associated constraint. Therefore, both tensor modes are physical degrees of freedom, and their Hamiltonian is exactly that of GR (see for example [18). In the vector case, $F^{i}$ is the only dynamical degree, while $S^{i}$ appears as a Lagrange multiplier, enforcing the constraints $\Pi_{F}^{i}=0$. These constraints are first class since they commute among each other and with the Hamiltonian $\mathcal{H}_{v}$, thus they represent the two gauge modes associated with vector perturbations. In summary, the tensor modes as the same as in GR and there are no physical vector degrees of freedom.

\section{References}

[1] M. Fierz and W. Pauli, Proc. Roy. Soc. Lond. A 173 (1939) 211.

[2] D. G. Boulware and S. Deser, Phys. Rev. D 6 (1972) 3368.

[3] K. Hinterbichler, arXiv:1105.3735 [hep-th].

[4] A. Salam and J. A. Strathdee, Phys. Rev. D 16 (1977) 2668.

[5] N. Arkani-Hamed, H. Georgi and M. D. Schwartz, Annals Phys. 305 (2003) 96 hep-th/0210184.

[6] V. F. Mukhanov and A. Wipf, Int. J. Mod. Phys. A 10 (1995) 579 hep-th/9401083.

[7] V. A. Rubakov and P. G. Tinyakov, Phys. Usp. 51, 759 (2008) arXiv:0802.4379 [hep-th]].

[8] S. Deser and A. Waldron, Phys. Lett. B 508 (2001) 347 hep-th/0103255.

[9] K. Koyama, G. Niz and G. Tasinato, Phys. Rev. Lett. 107 (2011) 131101 [arXiv:1103.4708 [hep-th]].

[10] K. Koyama, G. Niz and G. Tasinato, Phys. Rev. D 84 (2011) 064033 arXiv:1104.2143 [hep-th]].

[11] K. Koyama, G. Niz and G. Tasinato, JHEP 1112 (2011) 065 [arXiv:1110.2618 [hep-th]].

[12] G. D'Amico, C. de Rham, S. Dubovsky, G. Gabadadze, D. Pirtskhalava and A. J. Tolley, Phys. Rev. D 84, 124046 (2011) [arXiv:1108.5231 [hep-th]].

[13] A. E. Gumrukcuoglu, C. Lin and S. Mukohyama, JCAP 1111, 030 (2011) arXiv:1109.3845 [hep-th]].

[14] A. E. Gumrukcuoglu, C. Lin and S. Mukohyama, JCAP 1203 (2012) 006 [arXiv:1111.4107 [hep-th]].

[15] M. Crisostomi, D. Comelli and L. Pilo, arXiv:1202.1986 [hep-th].

[16] C. de Rham, G. Gabadadze and A. J. Tolley, Phys. Rev. Lett. 106, 231101 (2011) arXiv:1011.1232 [hep-th]].

[17] C. Aragone and J. Chela-Flores, Nuovo Cim. A 10, 818 (1972).

[18] J. -O. Gong, S. Koh and M. Sasaki, Phys. Rev. D 81 (2010) 084053 [arXiv:1002.1429 [hep-th]]. 